\title{
A splice acceptor site mutation in $T a G W 2-A 1$ increases thousand grain weight in tetraploid and hexaploid wheat through wider and longer grains
}

\author{
James Simmonds ${ }^{1} \cdot$ Peter $_{\text {Scott }^{1}} \cdot$ Jemima Brinton $^{1} \cdot$ Teresa C. Mestre $^{1,4} \cdot$ Max Bush $^{1} \cdot$ \\ Alicia del Blanco ${ }^{2} \cdot$ Jorge Dubcovsky $^{2,3} \cdot$ Cristobal Uauy $^{1}$ (i)
}

Received: 30 November 2015 / Accepted: 23 January 2016 / Published online: 16 February 2016

(C) The Author(s) 2016. This article is published with open access at Springerlink.com

\begin{abstract}
Key message Across 13 experiments the gw2-A1 mutant allele shifts grain size distribution consistently across all grains significantly increasing grain weight $(6.6 \%)$, width $(2.8 \%)$ and length $(2.1 \%)$ in tetraploid and hexaploid wheat.

Abstract There is an urgent need to identify, understand and incorporate alleles that benefit yield in polyploid wheat. The rice $O s G W 2$ gene functions as a negative regulator of grain weight and width and is homologous to the wheat $T a G W 2$ gene. Previously it was shown that transcript levels of the A-genome homoeologue, TaGW2-A1, are negatively associated with grain width in hexaploid wheat. In this study we screened the tetraploid Kronos TILLING population to identify mutants in TaGW2-A1. We identified a $\mathrm{G}$ to $\mathrm{A}$ transition in the splice acceptor site of exon 5 which leads to mis-splicing in TaGW2-A1. We backcrossed the mutant allele into tetraploid and hexaploid wheat and
\end{abstract}

Communicated by B. Keller.

Electronic supplementary material The online version of this article (doi:10.1007/s00122-016-2686-2) contains supplementary material, which is available to authorized users.

Cristobal Uauy

cristobal.uauy@jic.ac.uk

1 John Innes Centre, Norwich Research Park, Norwich NR4 7UH, UK

2 University of California Davis, One Shields Avenue, Davis, CA 95616, USA

3 Howard Hughes Medical Institute, Chevy Chase, MD 20815, USA

4 Present Address: CEBAS-CSIC, Espinardo, P.O. Box 164, 30100 Murcia, Spain generated a series of backcross derived isogenic lines which were evaluated in glasshouse and field conditions. Across 13 experiments the GW2-A1 mutant allele significantly increased thousand grain weight $(6.6 \%)$, grain width $(2.8 \%)$ and grain length $(2.1 \%)$ in tetraploid and hexaploid wheat compared to the wild type allele. In hexaploid wheat, this led to an increase in spike yield since no differences were detected for spikelet or grain number between isogenic lines. The increase in grain width and length was consistent across grains of different sizes, suggesting that the effect of the mutation is stable across the ear and within spikelets. Differences in carpel size and weight between alleles were identified as early as 5 days before anthesis, suggesting that TaGW2-A1 acts on maternal tissue before anthesis to restrict seed size. A single nucleotide polymorphism marker was developed to aid the deployment of the mutant allele into breeding programmes.

\section{Introduction}

Wheat provides approximately $20 \%$ of the calories and $25 \%$ of protein consumed by humankind (FAOSTAT 2015) and is an important source of micronutrients. There are two major types of polyploid wheat; hexaploid wheat $(2 n=6 x=42 ; \sim 17 \mathrm{Mb}$; AABBDD genomes) primarily used for bread and biscuit products and tetraploid durum wheat $(2 n=4 x=28 ; \sim 12 \mathrm{Mb}$; AABB genomes) used for pasta. The three genomes (A, B, and D referred to as homoeologues), have a complementary set of collinear genes that share between 96 and $98 \%$ sequence identity across coding regions (IWGSC 2014; Krasileva et al. 2013). Since most wheat genes are present as two or three close homoeologous copies (IWGSC 2014), mutations in individual genes can be masked by functional 
complementation by homoeologous genes present in the other genomes (Borrill et al. 2015; Lawrence and Pikaard 2003). This gene redundancy has limited the use of forward genetic screens in wheat and limited the detection of phenotypic effects of natural variants.

Despite a growing human population and rising demand for wheat, low rates of genetic gains for wheat yields (Ray et al. 2013) pose a real threat to global food security. Therefore there is an urgent need to identify, understand and incorporate alleles that increase wheat yield potential across multiple environments. This task is complicated by the polygenic regulation of grain yield and the strong effect of the environment. To facilitate its study, overall grain yield can be broken down into its individual components. This study focuses on thousand grain weight (TGW) and its physical parameters (grain length, width, area), which are inherited in a relatively stable manner and show higher heritability values than overall yield (Kuchel et al. 2007).

Significant advances have been made in our understanding of the genes controlling seed size in the model species rice (reviewed in Xing and Zhang 2010) and Arabidopsis (reviewed in $\mathrm{Li}$ and $\mathrm{Li}$ 2015). The characterised genes have been shown to function in G-protein signalling (Huang et al. 2009), phytohormone biosynthesis/signalling (Jiang et al. 2013), or in the ubiquitin-proteasome pathway ( $\mathrm{Li}$ et al. 2008; Song et al. 2007; Xia et al. 2013). Specifically in rice, genes with large effects on grain size have been identified revealing an independent genetic control of grain length and width in this species (Fan et al. 2006; Weng et al. 2008). This differs from our limited understanding in polyploid wheat where quantitative trait loci (QTL) for grain size and shape have been identified (Breseghello and Sorrells 2007; Maphosa et al. 2014; Rustgi et al. 2013; Simmonds et al. 2014; Sun et al. 2009; Williams and Sorrells 2014; Zhang et al. 2010), but no gene affecting TGW has yet been cloned.

The rice Grain Width and Weight 2 (OsGW2) gene encodes a previously unknown RING-type E3 ubiquitin ligase and functions as a negative regulator of grain width and weight in rice (Song et al. 2007). The wheat genome has three $G W 2$ homoeologues, with the A genome copy (TaGW2-A1) being most closely examined to date. Two studies have identified a significant association between a $T a G W 2-A 1$ promoter single nucleotide polymorphism (SNP) and TGW and grain width in Chinese germplasm (Su et al. 2011; Zhang et al. 2013). Despite discrepancies in the haplotype analysis, both studies show a negative relationship between $T a G W 2-A 1$ transcript levels and grain width. A separate study identified a single base insertion in the last exon of TaGW2-Al which results in a truncated protein. This allele was associated with increased TGW and grain width in hexaploid wheat compared to the full length wild type allele in a $F_{2: 3}$ population (Yang et al. 2012).
These results support a conserved role of $G W 2$ between wheat and rice as a negative regulator of grain size and weight. However, contradictory results have been reported from RNA interference (RNAi) studies of all three $T a G W 2$ homoeologues in wheat: whereas Bednarek et al. (2012) reported a reduction in grain weight and size by down-regulation of $T a G W 2$ homoeologues, Hong et al. (2014) found an increase in equivalent transgenic lines.

TILLING (Targeted Induced Local Lesion IN Genomes) is a powerful reverse genetics approach to characterise gene function in plants (McCallum et al. 2000). Both tetraploid and hexaploid TILLING populations are available in wheat (Uauy et al. 2009) and have been used to characterise genes involved in flowering time (Chen and Dubcovsky 2012; Chen et al. 2014), disease resistance (Fu et al. 2009) and end-use quality traits (Botticella et al. 2011; Hazard et al. 2012, 2014; Slade et al. 2012). The possibility of generating single, double and triple mutant combinations allows researchers to define contributions of specific homoeologues which can be informative for the deployment of mutant alleles for breeding.

In this study we screened a tetraploid TILLING population to identify mutants in TaGW2-A1. We identified a mutation in a splice acceptor site which leads to mis-splicing in exon 5 of TaGW2-A1. We backcrossed the mutant allele into tetraploid and hexaploid wheat and generated a series of BC-derived lines which were evaluated in both glasshouse and field conditions. The mutant allele consistently increased grain weight and morphometric parameters in all environments and across all grains of the spike compared to the wild type full length $T a G W 2-A 1$ allele. The effect was detected as early as 5 days before anthesis in developing carpels suggesting that GW2 acts on maternal tissue before anthesis to restrict seed size in wheat.

\section{Materials and methods}

\section{TILLING of TaGW2-A1}

We first used the $4545 x$ raw data of hexaploid wheat Chinese Spring (Brenchley et al. 2012) to reassemble the TaGW2-A1 gene region. These assemblies were combined with wheat ESTs to design primers in the introns flanking TaGW2-Al exons 2 and 6 (primers T1-T6). A touchdown PCR programme was used to amplify genomic DNA of Kronos and PCR products were cloned directly using a pGEM-T Easy kit (Promega, Southampton, UK) following the manufacturers' instructions. Miniprep DNA of clones were insert-sequenced by The Genome Analysis Centre (TGAC) using M13 primers. Sequence reads were aligned and assigned to the $\mathrm{A}$ genome using the sequence of the flow sorted chromosome arm DNA (Vrána et al. 
2000) and the IWGSC survey sequence which became publicly available afterwards (IWGSC 2014). Homoeologous SNPs between the A and B genomes were used to design a 6AS-specific forward primer (T12) to amplify a region of 1269-bp including exons 2-6 (amplicon T12-T6; GenBank accession KP749901) (Simmonds et al. 2014). Primers T12 and T6 were used to screen the first three plates of the tetraploid ethyl methane sulphonate (EMS) TILLING population using previously published protocols (Uauy et al. 2009). From the 20 putative mutants, a homozygous $\mathrm{G}$ to $\mathrm{A}$ transition in the AG splice acceptor site of exon 5 was identified in Kronos line T4-2235 (GenBank accession KP749902).

Three seeds of Kronos T4-2235 were germinated and genomic DNA was extracted from each plant (2235-1, -2, and -3) (Pallotta et al. 2003). The TaGW2-Al gene was cloned using primers T12 and T6 and GoTaq Mastermix (Promega, Southampton, UK), and $50 \mu \mathrm{l}$ PCR reactions were performed using a touch-down programme (66$61^{\circ} \mathrm{C}$ ) for all three plants. PCR products were checked by agarose electrophoresis and the single specific band from each PCR was excised and purified using a gel extraction kit (Qiagen, Manchester, UK). Sequencing was performed at TGAC using BigDye Terminator v3.1 Cycle Sequencing Kit (Life Technologies, Paisley, UK).

\section{Splicing of TaGW2-A1 transcripts in T4-2235 plants}

Leaf tips from T4-2235-1, -2, and -3, and wild type Kronos plants were harvested and total RNA isolated using TRIzol (Life Technologies). cDNAs were made using $3 \mu \mathrm{g}$ of total RNA in M-MLV Reverse Transcriptase reactions (Life Technologies). $0.5 \mu 1$ of cDNA was cloned using primers MF1 and MR1 (Table S1), GoTaq Mastermix and a touchdown programme $\left(65-60^{\circ} \mathrm{C}\right)$. PCR products were ligated into a TOPO vector (Life Technologies), heat-shocked into One Shot E.coli cells (Life Technologies) and colonies selected on Spectinomycin LB agar media. DNA from surviving colonies was sequenced at TGAC.

Quantitative reverse-transcription PCR (qRT-PCR) was performed on developing grains and flag leaves of a $\mathrm{BC}_{4}$ near isogenic line (NIL) of Kronos carrying the G2373A mutation. RNA and cDNA was made as described above. Transcript levels were determined using forward primers which were designed to specifically amplify the $-4 \mathrm{bp}$ and -9 bp transcript of $T a G W 2-A$, and a common reverse primer (Online Resource 1). Primer efficiencies for the -4 bp (1.04) and -9 bp (1.09) assay were determined using the cDNA from the mutant NIL. qRT-PCR reactions were carried out with a LightCycler 480 instrument (Roche Applied Science, UK) using LightCycler 480 SYBR Green I Master Mix (Roche Applied Science, UK) and the following conditions: $5 \mathrm{~min}$ at $95{ }^{\circ} \mathrm{C} ; 40$ cycles of $15 \mathrm{~s}$ at $95{ }^{\circ} \mathrm{C}, 15 \mathrm{~s}$ at $60{ }^{\circ} \mathrm{C}, 20 \mathrm{~s}$ at $72{ }^{\circ} \mathrm{C}$. The specificity of the amplicon was determined by dissociation curve analysis (from 60 to $95{ }^{\circ} \mathrm{C}$ ). Transcript levels were normalized with actin (Uauy et al. 2006) and linearized values determined using the $(1+\text { Efficiency) })^{-\Delta \Delta C T}$ method (Schmittgen and Livak 2008). Five biological replicates of both flag leaves and developing grains 15 days post anthesis were used in the analysis and the results were averaged across two independent technical replicates.

\section{KASPar development}

A KASP assay targeting the G2373A SNP was developed (K1-K2/K3; Online Resource 1 and 2) and validated in the three T4-2235 plants. Briefly, two allele specific reverse primers were designed for the G2373A SNP incorporating either the $\mathrm{G}$ or $\mathrm{A}$ polymorphism at the $3^{\prime}$ end (primer K2 for the wildtype (G) and $\mathrm{K} 3$ for the mutant (A) allele). The common primer was developed to discriminate against the B genome by utilising a $3 \mathrm{bp}$ indel present in the A genome (primer K1). Allele specific primers were synthesised with FAM and HEX tails and reactions and cycling conditions were as described before (Trick et al. 2012). Fluorescent end-point genotyping was carried out using a Tecan Safire plate reader (Tecan Group AG, Mannerdorf, Switzerland).

\section{Plant growth and NIL development}

TILLING mutant T4-2235 plant 3 was crossed to Kronos and the resulting $F_{1}$ self-pollinated to produce a segregating $\mathrm{F}_{2}$ population. NILs were developed through subsequent backcrossing to Kronos using the K1-K2/K3 KASPar marker for selection. At both the $\mathrm{BC}_{2}$ and $\mathrm{BC}_{4}$ generations heterozygous plants were self-pollinated and homozygous NILs extracted from the resultant $\mathrm{BC}_{2} \mathrm{~F}_{2}$ and $\mathrm{BC}_{4} \mathrm{~F}_{2}$ plants. A similar backcrossing scheme was followed for the introgression of the mutation into hexaploid wheat, cv. Paragon.

Plants were grown in lit greenhouses with $16 \mathrm{~h}$ light (250-300 $\mu \mathrm{mol} \mathrm{m} \mathrm{m}^{-2} \mathrm{~s}^{-1}$ ) and $8 \mathrm{~h}$ dark at $18{ }^{\circ} \mathrm{C}$ and were sown in Petersfield Cereal Mix (Petersfield, Leicester, UK). In the greenhouse, experimental units (a single plant in a $1 \mathrm{~L}$ pot) were organized in a complete randomized design (CRD) for both the Kronos and Paragon germplasm. Field experiments were conducted in Norwich, UK $\left(52^{\circ} 37^{\prime} 39.9^{\prime \prime} \mathrm{N}, 1^{\circ} 10^{\prime} 45.9^{\prime \prime} \mathrm{E}\right)$ in 2013,2014 , and 2015 and Tulelake, CA, USA $\left(41^{\circ} 57^{\prime} 47.9^{\prime \prime} \mathrm{N}, 121^{\circ} 28^{\prime} 12.7^{\prime \prime} \mathrm{W}\right)$ in 2013, and organized as either CRD (2013-2014) or randomized complete block designs (RCBD; 2015). Experimental units in the field consisted of $1 \mathrm{~m}$ rows $\left(\mathrm{F}_{3}\right)$ or $\sim 6.6 \mathrm{~m}^{2}$ plots $\left(\mathrm{BC}_{2}\right.$ and $\mathrm{BC}_{4}$ NILs) depending on the germplasm being studied. 


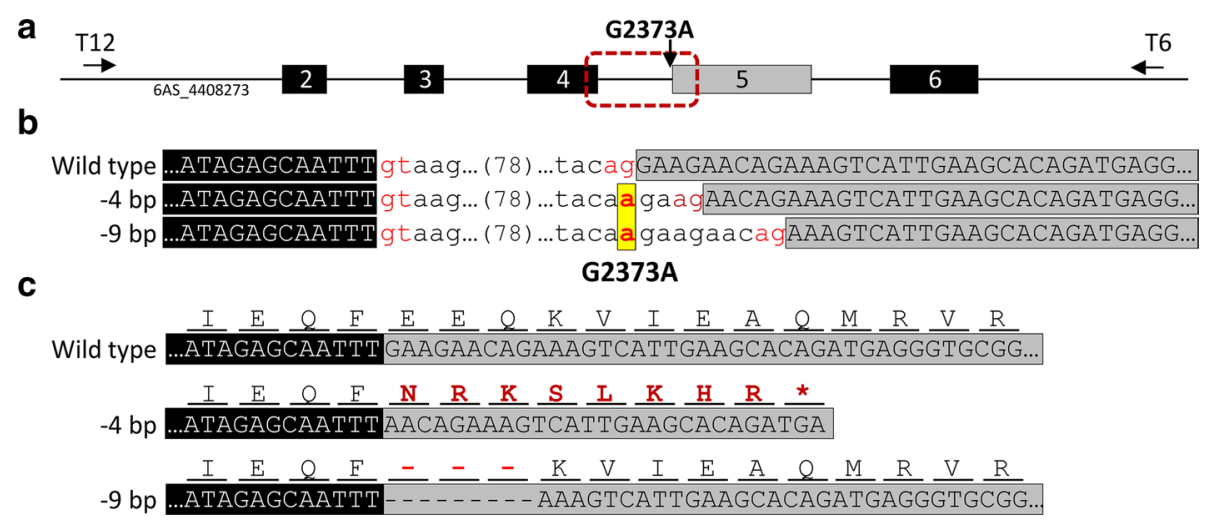

Fig. 1 G2373A results in mis-splicing of TaGW2-A1. a Diagram of TaGW2-A1 target region including exons 2-6 (black and grey numbered boxes) and introns (thin line). The position of the G $>$ A transition at position 2373 (G2373A) of IWGSC_CSS_6AS_ scaff_4408273 is indicated. b Sequence alignment of gDNA from wild type (top) and mutant line T4-2235 (middle and bottom) which includes the G2373A transition. The G2373A mutation in line T4-2235 is in red font and yellow highlight. Exon sequences are in uppercase letters (exon 4 in black; exon 5 in grey), whereas intron sequences adjacent to the splice sites (red font) are in lowercase. The 78-bp of intron four are represented by the number 78 between

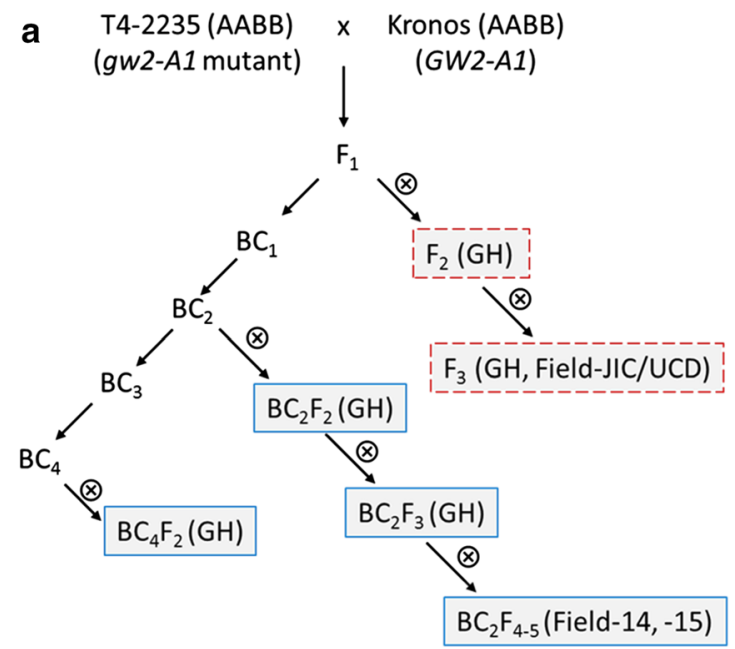

Fig. 2 Crossing scheme used to introduce the G2373A allele ( $g w 2$ A) into tetraploid wheat Kronos a and hexaploid wheat Paragon b both carrying a wild type GW2-A allele. Small crossed circles indicate self-pollination, whereas straight arrows indicate back-cross to either Kronos (a) or Paragon (b). For the Kronos stream (a), $\mathrm{F}_{2}$,

\section{Grain phenotyping}

Average grain morphometric measurements (grain width, length, area) and thousand grain weight were recorded using the MARVIN seed analyser (GTA Sensorik GmbH, Germany). In glasshouse experiments, values for individual plants were calculated from the means of 3 or 4 ears $\left(F_{2}\right.$ and $F_{3}$ experiments, respectively), or from all parentheses. Note that the mutation leads to a change in the AG splice acceptor site in T4-2235 which removes either four bp (GAAG) or nine bp (GAAGAACAG) from exon 5. c Nucleotide sequence of cDNA spanning exons 4 and 5 in wild type (top sequence) and the two variants of mutant T4-2235 (middle and bottom sequences), with their corresponding amino acid translations. The -4 bp mutant allele is missing four nucleotides which disrupts the reading frame (red amino acid residues) leading to a premature termination codon (red asterisk) in the T4-2235 sequence. The -9 bp mutant allele is missing nine nucleotides which leads to the loss of three amino acids (EEQ) from the mutant protein

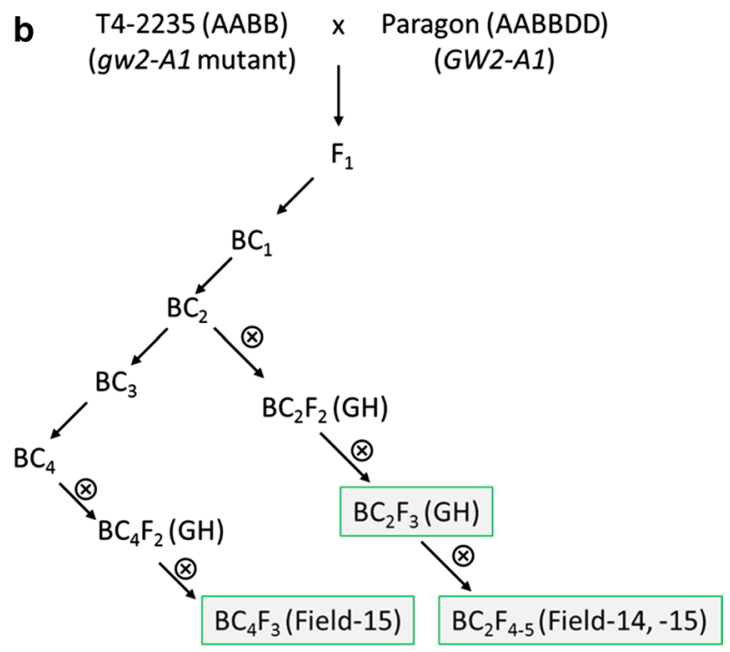

$\mathrm{BC}_{2^{-}}$, and $\mathrm{BC}_{4}$-derived lines were evaluated under glasshouse $(\mathrm{GH})$ and field environments, whereas for the Paragon stream (b) only $\mathrm{BC}_{2}-$ and $\mathrm{BC}_{4}$-derived lines were evaluated. The results of each generation are indicated by the outline colour (red dash: Table 1; blue: Table 2; Fig. 4a; green Tables 3, 4; Fig. 4b)

available harvested ears (BC NILs). Values from field experiments were based on a single sample of approximately 400 grains from each experimental unit $(1 \mathrm{~m}$ row or $6.6 \mathrm{~m}^{2}$ plot). These average values were used in the statistical analyses below. In addition, raw individual grain data for each BC-derived plant was extracted and all grains within the same genotypic class were grouped to generate box-and-whisker plots. The objective of this last 
Table 1 Effect of G2373A gw2-A1 allele on thousand grain weight (TGW) and grain morphometric parameters in $\mathrm{F}_{2}$ and $\mathrm{F}_{3}$ populations of tetraploid wheat Kronos

\begin{tabular}{|c|c|c|c|c|c|c|}
\hline Experiment & Genotype (allele) & $N$ & TGW (g) & Area $\left(\mathrm{mm}^{2}\right)$ & Width (mm) & Length (mm) \\
\hline \multirow{4}{*}{$\begin{array}{l}\mathrm{F}_{2} \\
\mathrm{GH}\end{array}$} & $G W 2-A 1 / G W 2-A 1$ (Kronos) & 18 & $50.4 \pm 1.4$ & $21.7 \pm 0.3$ & $3.73 \pm 0.04$ & $7.47 \pm 0.05$ \\
\hline & $G W 2-A 1 / g w 2-A 1$ (heterozygous) & 53 & $54.1 \pm 0.8$ & $22.7 \pm 0.2$ & $3.83 \pm 0.02$ & $7.65 \pm 0.03$ \\
\hline & $g w 2-A 1 / g w 2-A 1(\mathrm{G} 2373 \mathrm{~A})$ & $18^{\mathrm{a}}$ & $55.8 \pm 1.7$ & $23.1 \pm 0.4$ & $3.83 \pm 0.04$ & $7.73 \pm 0.06$ \\
\hline & & & $10.6 \%{ }^{*, \mathrm{~b}}$ & $6.8 \% * *$ & $2.5 \%$ & $3.4 \% * *$ \\
\hline \multicolumn{7}{|l|}{$\mathrm{F}_{3}$} \\
\hline \multirow[t]{3}{*}{ GH } & $G W 2-A 1 / G W 2-A 1$ (Kronos) & 78 & $29.5 \pm 0.6$ & $18.9 \pm 0.1$ & $3.22 \pm 0.02$ & $7.33 \pm 0.02$ \\
\hline & $g w 2-A 1 / g w 2-A 1(\mathrm{G} 2373 \mathrm{~A})$ & 80 & $30.6 \pm 0.7$ & $19.5 \pm 0.2$ & $3.28 \pm 0.02$ & $7.43 \pm 0.03$ \\
\hline & & & $3.8 \%$ & $3.2 \% * *$ & $2.0 \% *$ & $1.5 \% * *$ \\
\hline \multirow[t]{3}{*}{ Field-JIC } & $G W 2-A 1 / G W 2-A 1$ (Kronos) & 18 & $36.3 \pm 0.8$ & $20.1 \pm 0.2$ & $3.42 \pm 0.02$ & $7.3 \pm 0.06$ \\
\hline & $g w 2-A 1 / g w 2-A 1(\mathrm{G} 2373 \mathrm{~A})$ & 19 & $39.3 \pm 1.1$ & $21 \pm 0.2$ & $3.52 \pm 0.03$ & $7.43 \pm 0.06$ \\
\hline & & & $8.2 \% *$ & $4.7 \% * *$ & $2.7 \% *$ & $1.8 \%$ \\
\hline \multirow[t]{3}{*}{ Field-UCD } & $G W 2-A 1 / G W 2-A 1$ (Kronos) & 18 & $55.5 \pm 0.7$ & $22.9 \pm 0.2$ & $3.72 \pm 0.02$ & $7.73 \pm 0.04$ \\
\hline & $g w 2-A 1 / g w 2-A 1(\mathrm{G} 2373 \mathrm{~A})$ & 19 & $57.4 \pm 0.6$ & $23.7 \pm 0.2$ & $3.74 \pm 0.02$ & $7.94 \pm 0.03$ \\
\hline & & & $3.5 \%$ & $3.5 \% * *$ & $0.6 \%$ & $2.7 \% * * *$ \\
\hline \multicolumn{3}{|c|}{ Overall ANOVA $\mathrm{F}_{3}$ populations ( $P$ value) } & 0.023 & $<0.001$ & 0.002 & $<0.001$ \\
\hline
\end{tabular}

Four experiments were conducted across glasshouse $(\mathrm{GH})$ and field environments. $N$ corresponds to number of plants $(\mathrm{GH})$ or $1 \mathrm{~m}$ rows (field). The overall ANOVA was conducted for the three $\mathrm{F}_{3}$ experiments

$*(P<0.05), * *(P<0.01)$, *** $(P<0.001)$

a In the $\mathrm{F}_{2}$ experiment, phenotypic data from a single homozygous mutant line was ambiguously labelled and therefore excluded. This line was recovered in the $\mathrm{F}_{3}$ experiments and confirmed through the KASP marker

b The percentages correspond to the difference between the $g w 2-A 1$ and GW2-A1 phenotypes as a percentage of the GW2-A1 wild type Kronos allele

Table 2 Effect of G2373A gw2-A1 allele on thousand grain weight (TGW) and grain morphometric parameters in tetraploid $\mathrm{BC}_{2}$ and $\mathrm{BC}_{4}$ lines

\begin{tabular}{|c|c|c|c|c|c|c|}
\hline Experiment & Genotype (allele) & $N$ & TGW (g) & Area $\left(\mathrm{mm}^{2}\right)$ & Width (mm) & Length (mm) \\
\hline \multirow[t]{3}{*}{$\mathrm{BC}_{2} \mathrm{~F}_{2}(\mathrm{GH})$} & $G W 2-A 1 / G W 2-A 1$ (Kronos) & 15 & $51.4 \pm 0.8$ & $23.6 \pm 0.2$ & $3.91 \pm 0.02$ & $7.79 \pm 0.03$ \\
\hline & $g w 2-A 1 / g w 2-A 1(\mathrm{G} 2373 \mathrm{~A})$ & 10 & $55.2 \pm 1.3$ & $24.8 \pm 0.2$ & $4.06 \pm 0.03$ & $7.93 \pm 0.04$ \\
\hline & & & $7.5 \% * *, \mathrm{a}$ & $5.2 \% * *$ & $3.8 \% * *$ & $1.8 \% * *$ \\
\hline \multirow[t]{3}{*}{$\mathrm{BC}_{2} \mathrm{~F}_{3}(\mathrm{GH})$} & $G W 2-A 1 / G W 2-A 1$ (Kronos) & 121 & $50.5 \pm 0.4$ & $22.2 \pm 0.1$ & $3.95 \pm 0.01$ & $7.21 \pm 0.02$ \\
\hline & $g w 2-A 1 / g w 2-A 1(\mathrm{G} 2373 \mathrm{~A})$ & 110 & $53.2 \pm 0.4$ & $23.2 \pm 0.1$ & $4.08 \pm 0.01$ & $7.32 \pm 0.02$ \\
\hline & & & $5.4 \% * * *$ & $4.4 \% * * *$ & $3.2 \% * * *$ & $1.6 \% * * *$ \\
\hline \multirow[t]{3}{*}{$\mathrm{BC}_{4} \mathrm{~F}_{2}(\mathrm{GH})$} & $G W 2-A 1 / G W 2-A 1$ (Kronos) & 18 & $72.3 \pm 1.3$ & $27.5 \pm 0.3$ & $4.28 \pm 0.03$ & $8.32 \pm 0.05$ \\
\hline & $g w 2-A 1 / g w 2-A 1(\mathrm{G} 2373 \mathrm{~A})$ & 16 & $75.5 \pm 1.2$ & $29.2 \pm 0.3$ & $4.42 \pm 0.03$ & $8.57 \pm 0.05$ \\
\hline & & & $4.4 \%$ & $6.1 \% * *$ & $3.1 \% *$ & $3.0 \% * *$ \\
\hline \multirow[t]{3}{*}{$\mathrm{BC}_{2} \mathrm{~F}_{4}$ (Field-14) } & $G W 2-A 1 / G W 2-A 1$ (Kronos) & 11 & $51.3 \pm 0.9$ & $23.0 \pm 0.2$ & $3.75 \pm 0.02$ & $7.76 \pm 0.03$ \\
\hline & $g w 2-A 1 / g w 2-A 1(\mathrm{G} 2373 \mathrm{~A})$ & 8 & $54.9 \pm 0.8$ & $24.2 \pm 0.3$ & $3.88 \pm 0.03$ & $7.96 \pm 0.06$ \\
\hline & & & $7.0 \% * *$ & $5.5 \% * * *$ & $3.5 \% * *$ & $2.6 \% * *$ \\
\hline \multirow[t]{3}{*}{$\mathrm{BC}_{2} \mathrm{~F}_{5}$ (Field-15) } & $G W 2-A 1 / G W 2-A 1$ (Kronos) & 15 & $58.7 \pm 1.0$ & $22.9 \pm 0.2$ & $3.60 \pm 0.03$ & $8.32 \pm 0.03$ \\
\hline & $g w 2-A 1 / g w 2-A 1(\mathrm{G} 2373 \mathrm{~A})$ & 18 & $62.5 \pm 0.8$ & $24.1 \pm 0.3$ & $3.73 \pm 0.02$ & $8.47 \pm 0.03$ \\
\hline & & & $6.4 \% * *$ & $5.5 \% * *$ & $3.7 \% * *$ & $1.7 \% * *$ \\
\hline \multicolumn{3}{|c|}{ Overall ANOVA BC populations ( $P$ value) } & $<0.001$ & $<0.001$ & $<0.001$ & $<0.001$ \\
\hline
\end{tabular}

Experiments were conducted across glasshouse (GH) and field environments (2014 and 2015). N corresponds to number of plants (GH) or plots (field)

$*(P<0.05), * *(P<0.01), * * *(P<0.001)$

a The percentages correspond to the difference between the $g w 2-A 1$ and $G W 2-A 1$ phenotypes as a percentage of the GW2-A1 wild type Kronos allele 
Table 3 Effect of G2373A gw2-A1 allele on thousand grain weight (TGW) and grain morphometric parameters in hexaploid $\mathrm{BC}_{2}$ Paragon lines

\begin{tabular}{|c|c|c|c|c|c|c|}
\hline Experiment & Genotype (allele) & $N$ & TGW (g) & Area $\left(\mathrm{mm}^{2}\right)$ & Width (mm) & Length (mm) \\
\hline \multirow[t]{3}{*}{$\mathrm{BC}_{2} \mathrm{~F}_{3}(\mathrm{GH})$} & $G W 2-A 1 / G W 2-A 1$ (Paragon) & 65 & $37.0 \pm 0.3$ & $18.8 \pm 0.1$ & $3.74 \pm 0.01$ & $6.16 \pm 0.01$ \\
\hline & $g w 2-A 1 / g w 2-A 1(\mathrm{G} 2373 \mathrm{~A})$ & 58 & $39.7 \pm 0.4$ & $19.5 \pm 0.1$ & $3.84 \pm 0.01$ & $6.26 \pm 0.03$ \\
\hline & & & $7.1 \% * * *, \mathrm{a}$ & $4.1 \% * * *$ & $2.6 \% * * *$ & $1.6 \% * * *$ \\
\hline \multirow[t]{3}{*}{$\mathrm{BC}_{2} \mathrm{~F}_{4}($ Field-14) } & GW2-A1/GW2-A1 (Paragon) & 10 & $43.8 \pm 0.3$ & $20.5 \pm 0.1$ & $3.76 \pm 0.01$ & $6.63 \pm 0.01$ \\
\hline & $g w 2-A 1 / g w 2-A 1(\mathrm{G} 2373 \mathrm{~A})$ & 8 & $46.9 \pm 0.3$ & $21.4 \pm 0.1$ & $3.89 \pm 0.01$ & $6.73 \pm 0.03$ \\
\hline & & & $7.0 \% * * *$ & $4.1 \% * * *$ & $3.4 \% * * *$ & $1.6 \% * *$ \\
\hline \multirow[t]{3}{*}{$\mathrm{BC}_{2} \mathrm{~F}_{5}($ Field-15) } & $G W 2-A 1 / G W 2-A 1$ (Paragon) & 10 & $44.5 \pm 0.8$ & $17.9 \pm 0.2$ & $3.4 \pm 0.02$ & $6.45 \pm 0.03$ \\
\hline & $g w 2-A 1 / g w 2-A 1(\mathrm{G} 2373 \mathrm{~A})$ & 10 & $47.0 \pm 0.8$ & $18.5 \pm 0.1$ & $3.48 \pm 0.02$ & $6.53 \pm 0.03$ \\
\hline & & & $5.6 \% * * *$ & $3.3 \% * * *$ & $2.4 \% * * *$ & $1.3 \%$ \\
\hline \multirow[t]{3}{*}{$\mathrm{BC}_{4} \mathrm{~F}_{3}$ (Field-15) } & GW2-A1/GW2-A1 (Paragon) & 44 & $47.4 \pm 0.3$ & $19.1 \pm 0.1$ & $3.62 \pm 0.01$ & $6.52 \pm 0.01$ \\
\hline & $g w 2-A 1 / g w 2-A 1(\mathrm{G} 2373 \mathrm{~A})$ & 40 & $51.9 \pm 0.5$ & $20.3 \pm 0.1$ & $3.73 \pm 0.01$ & $6.69 \pm 0.03$ \\
\hline & & & $9.4 \% * * *$ & $5.9 \% * * *$ & $3.0 \% * * *$ & $2.7 \% * * *$ \\
\hline \multicolumn{3}{|c|}{ Overall ANOVA BC populations ( $P$ value) } & $<0.001$ & $<0.001$ & $<0.001$ & $<0.001$ \\
\hline
\end{tabular}

Experiments were conducted in glasshouse (GH) and field environments (2014 and 2015). N corresponds to number of plants (GH) or plots (field)

$* *(P<0.01), * * *(P<0.001)$

a The percentages correspond to the difference between the $g w 2-A 1$ and $G W 2-A 1$ phenotypes as a percentage of the GW2-A1 wild type Paragon allele

analysis was to show the effect of the $T a G W 2-A 1$ gene on grains of different sizes.

\section{Carpel/grain developmental time course}

Paragon $\mathrm{BC}_{2}$ NILs grown in Norwich in 2014-2015 were used for the carpel/grain developmental time course. These genotypes included two independent $\mathrm{BC}_{2}$ NILs with the G2373A mutation and two sibling NILs with the wild type Paragon allele. In each of four blocks, we tagged 65 ears per genotype at ear emergence (peduncle just visible) on the same day to ensure that subsequent sampling took place at the same developmental stage. Ten spikes per genotype (per block) were sampled at six time-points: heading ( -5 days post anthesis), anthesis (0 dpa), 3, 9, 16, and $23 \mathrm{dpa}$. From these spikes, ten carpels/grains were sub-sampled from the two outer florets (floret positions F1 and F2) of the five spikelets located in the middle of the spike. The carpels/ grains were then weighed (fresh weight), morphometric parameters measured on the MARVIN analyser, and then dried to constant dry weight in an oven at $37{ }^{\circ} \mathrm{C}$. In total $\sim 100$ carpels/grains were sampled per block for each genotype at each time-point (10 spikes $\times 10$ carpels/grains). For the statistical analysis, however, the carpels/grains and spikes were considered as sub-samples of the experimental unit and hence the average of the individual genotype within the block was used (i.e. the average of 100 carpels/grains).

\section{Statistical analysis}

The Chi-squared test was performed using the CHITEST function in Excel (Microsoft Office, Microsoft) to evaluate the segregation ratios in the $\mathrm{F}_{2}$ Kronos glasshouse experiment. To assess the differences between the $G W 2-A 1$ and $g w 2-A 1$ alleles, the average grain morphometric parameters and TGW of experimental units were analysed using one-way (GH and Field CRD experiments) and two-way (Field RCBD experiments) analysis of variance (ANOVA). For the $\mathrm{F}_{2}$ glasshouse experiment, the effect of the TaGW2-Al gene was partitioned into additive (linear) and dominant (quadratic) effects using orthogonal contrasts. The three $\mathrm{F}_{3}$ experiments were jointly analysed in a two-way ANOVA with the $T a G W 2-A 1$ allele and the experiment included in the model (Table 1). Similar two-way ANOVAs were conducted for the five tetraploid BC-derived experiments (Table 2) and the four hexaploid experiments (Table 3). For the carpel/grain time course, a two-way ANOVA including genotype and block was used for the analysis. Differences in expression levels between mutant transcripts were assessed by ANOVA using the technical replicates as a block and the transcript, tissue, and transcript*tissue interaction in the model. ANOVAs were performed using GenStat 15th edition (http:// www.vsni.co.uk/). 


\section{Results}

\section{Identification and characterisation of the TaGW2-AI mutant}

Primers T12 and T6 were used to screen a region of 1269bp including exons 2-6 in 1152 individuals of the Kronos EMS TILLING population. Among the 20 putative mutants identified within this region, we found a homozygous mutation in the guanine residue of the AG canonical splice acceptor site of exon 5 in Kronos TILLING line T4-2235 (Fig. 1a). Using the recently published wheat chromosome arm survey sequence as a reference (scaffold 6AS_4408273), the G-A transition is 2373-bp from the predicted start codon of $T a G W 2-A 1$ and was labelled as G2373A.

The homozygous mutation was confirmed in three independent $\mathrm{M}_{3}$ plants derived from $\mathrm{T} 4-2235$, all showing the G2373A transition. No additional mutations were found across the TaGW2-Al coding sequence in T4-2235 plants. A genome-specific KASP assay was developed to facilitate the selection of the mutant G2373A allele during subsequent population development and in marker assisted selection (K1-K2/K3; Online Resource 2).

Several potential alternative AG splice acceptor sites were identified in exon 5 immediately following the EMS mutation. Therefore, we sequenced cDNA from leaves of T4-2235 plants to determine the effect of the G2373A splice site mutation on the TaGW2-A1 transcript. Based on the sequencing of 12 independent clones we found that in nine clones the splicing happened at the AG located 4-bp downstream of the wild type splice site, whereas for three clones splicing occurred after the AG located 9-bp from the wild type site. The -4 bp transcript (Fig. 1b) results in a frame-shift that generates a premature termination codon 27-bp within exon 5 (Fig. 1c). This results in the truncation of the C-terminus 290 amino acids and a shorter protein of only 134 amino acids. The $-9 \mathrm{bp}$ transcript results in a shorter, but in-frame, cDNA encoding a GW2 protein missing three amino acids (E127, E128, Q129). We further characterised the relative expression of the -4 and $-9 \mathrm{bp}$ transcripts through qRT-PCR of $\mathrm{BC}_{4}$ near-isogenic lines of the G2373A allele in the Kronos background. In both grain and leaf tissue we found 15-20-fold higher expression of the -4 bp transcript, compared to the -9 bp transcript $(P=0.019)$. No significant effect of tissue type or tissue*transcript interaction was detected. Thus, based on the cloning and expression data, the mis-splicing of exon 5 from the G2373A mutation results in two types of mutant protein; predominantly a 134 amino acid truncated GW2 protein, and less frequently a GW2 protein missing three amino acids.
The G2373A mutation leads to increased grain size in tetraploid wheat

To assess the effect of the G2373A mutation on grain morphometric parameters we crossed T4-2235 (plant 3) to wild type Kronos to generate segregating populations (Fig. 2a). We first evaluated an $F_{2}$ population of 90 individuals which were genotyped with the K1-K2/K3 KASP assay. We identified 18 homozygous plants for the Kronos wild type, 19 homozygous plants with the G2373A mutant allele, and 53 heterozygous individuals, consistent with the expected $1: 2: 1$ segregation ratio $\left(\chi^{2}=2.86 ; P=0.239\right)$. Homozygous plants with the G2373A mutant allele had significantly higher TGW $(10.6 \%, P=0.04)$ and larger grain area $(6.8 \%, P=0.006)$ than lines with the Kronos wild type allele (Table 1). This was due to a combined effect of wider and longer grains, although only grain length was statistically significant $(3.4 \%, P<0.01)$. The effect of the G2373A mutation was additive for TGW, grain area and length as indicated by the highly significant linear contrasts $(P \leq 0.01)$ and the non-significant quadratic contrasts $(P>0.27)$ for each of these traits.

To further assess this effect of G2373A on grain morphometric parameters, the homozygous $\mathrm{F}_{2}$ plants for Kronos and G2373A were self-pollinated and grown in three separate experiments. These included a replicated glasshouse experiment $\left(\mathrm{F}_{3}-\mathrm{GH}\right)$ and two field trials conducted in Norwich, UK (JIC) and Tulelake, CA (UCD). Table 1 describes the statistical analyses for the experiments separately and combined and for the varying contributions of grain length and width. Across all three experiments, $\mathrm{F}_{3}$ plants with the G2373A mutant allele had significantly larger TGW than wild type plants (ranging from 3.5 to $8.2 \%, P=0.023$ ), although within experiments this was only significant in the JIC field trials. Grain length and width were also significantly higher in mutant lines $(P<0.001$ and $P=0.002$, respectively), although under field conditions only one of these effects was significant in UCD (length) and JIC (width). Across experiments G2373A mutant plants had significantly larger grain area than wild type plants, ranging from 3.2 to $4.7 \%(P<0.001)$. These experiments on $\mathrm{F}_{3}$ plants provide further evidence of the significant effect of $T a G W 2-A 1$ on grain size parameters in tetraploid wheat, although the relative contributions to grain length and width vary across growing environments.

In addition to the G2373A mutation in TaGW2-A1, line T4-2235-3 is expected to carry over 2000 mutations in protein coding genes across its genome (Uauy et al. 2009). Therefore, we backcrossed the mutant allele into Kronos to reduce the mutation load and to evaluate the effect of this mutation in a more homogenous genetic background. We evaluated homozygous lines derived from this backcrossing 
Fig. 3 Representative grains from field grown $\mathrm{BC}_{2} \mathrm{~F}_{4}$ near isogenic lines carrying the wild type GW2-A1 or G2373A $g w 2$ A1 mutant allele in tetraploid wheat Kronos and hexaploid wheat Paragon. Grains are aligned to show differences in width (20 grains) and length (10 grains). Scale bar $=1 \mathrm{~cm}$

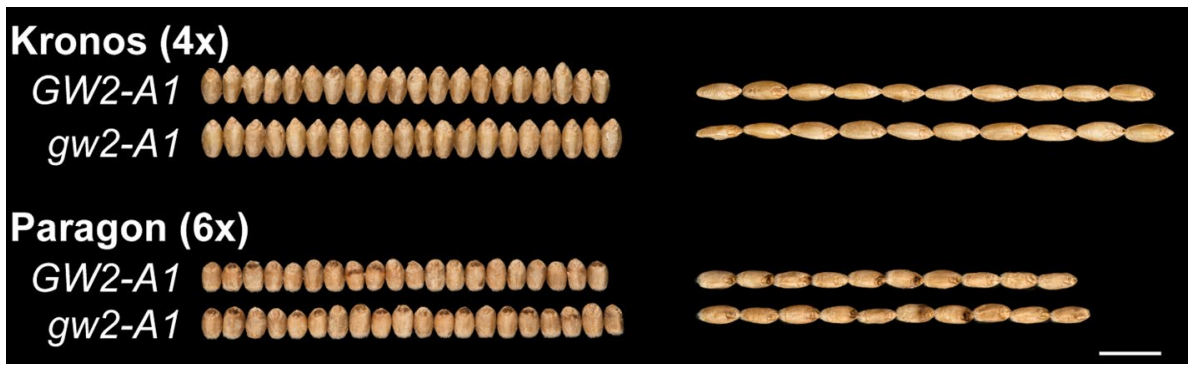

Table 4 Effect of G2373A allele on spike yield components in hexaploid $\mathrm{BC}_{2}$ and $\mathrm{BC}_{4}$ Paragon lines

\begin{tabular}{llllll}
\hline & GW2 allele & Spike yield & Spikelet number & Seeds/spike & Seeds/spikelet \\
\hline $\mathrm{BC}_{2} \mathrm{~F}_{5}$ (Field-15) & GW2-A1/GW2-A1 (Paragon) & $2.64 \pm 0.15$ & $18.3 \pm 0.4$ & $59.0 \pm 3.1$ & $3.21 \pm 0.13$ \\
& $g w 2-A 1 / g w 2-A 1$ (G2373A) & $2.71 \pm 0.07$ & $18 \pm 0.2$ & $57.6 \pm 1.1$ & $3.20 \pm 0.05$ \\
& & $2.7 \%^{\mathrm{a}}$ & $-1.8 \%$ & $-2.5 \%$ & $-0.4 \%$ \\
$\mathrm{BC}_{4} \mathrm{~F}_{3}$ (Field-15) & $G W 2-A 1 / G W 2-A 1$ (Paragon) & $3.25 \pm 0.04$ & $19.2 \pm 0.1$ & $68.5 \pm 0.8$ & $3.32 \pm 0.03$ \\
& $g w 2-A 1 / g w 2-A 1$ (G2373A) & $3.56 \pm 0.09$ & $19.5 \pm 0.1$ & $68.7 \pm 1.5$ & $3.32 \pm 0.06$ \\
& & $9.6 \% * * *$ & $1.2 \% *$ & $0.3 \%$ & $0.2 \%$ \\
Overall ANOVA BC populations $(P$ value) & $<0.001$ & 0.428 & 0.913 \\
\hline
\end{tabular}

Values are calculated from the same plants presented in Table 3

$*(P<0.05), * * *(P<0.001)$

a The percentages correspond to the difference between the $g w 2-A 1$ and $G W 2-A 1$ phenotypes as a percentage of the GW2-A1 wild type Paragon allele

scheme at the $\mathrm{BC}_{2}$ and $\mathrm{BC}_{4}$ stages in both $\mathrm{GH}\left(\mathrm{BC}_{2-4}\right)$ and field $\left(\mathrm{BC}_{2} ; 2014-2015\right)$ conditions (Fig. 2a). We identified consistent and highly significant effects of the G2373A mutation on TGW across all five experiments (ranging from 4.4 to $7.5 \% ; P<0.001$ ) (Table 2 ). This was due to significant effects on both grain width and length which increased on average $3.5 \%(P<0.001)$ and $2.2 \%(P<0.001)$, respectively, in lines carrying the G2373A gw2-A1 mutant allele. These combined effects led to an overall significant effect on grain area across experiments of $5.4 \%(P<0.001)$. Importantly, the effects on TGW and grain size were consistent across both glasshouse and field environments.

\section{The G2373A mutation increases TGW in hexaploid wheat}

To assess the effect of the G2373A mutation in hexaploid wheat we crossed the tetraploid T4-2235-3 mutant plant with hexaploid wheat cultivar Paragon (Fig. 2b). Pentaploid $\mathrm{F}_{1}$ plants were crossed again to Paragon and heterozygous $\mathrm{BC}_{1}$ individuals were selected using the K1-K2/K3 KASP assay and further backcrossed to Paragon. Experiments were conducted in glasshouse and field environments using homozygous $\mathrm{BC}_{2}$ and $\mathrm{BC}_{4}$ plants. Across the four environments, the G2373A mutation significantly increased all grain morphometric parameters studied compared to the wild type Paragon allele. The G2373A mutation increased grain area $(4.3 \% ; P<0.001)$, grain width $(2.9 \% ; P<0.001)$ and length $(1.8 \%, P<0.001)$ resulting in a consistent increase in TGW (7.3\%; $P<0.001$ ) (Table 3). The TaGW2-Al G2373A mutation thus has a consistent effect on the mean value for grain size parameters in both tetraploid and hexaploid wheat. Representative grains from field grown BC-derived lines of Kronos and Paragon are represented in Fig. 3.

We also evaluated the effect of the G2373A mutation on spike yield components in the field grown Paragon $\mathrm{BC}_{2}$ and $\mathrm{BC}_{4}$ lines in 2015. Across the two experiments we found no significant effects of the mutant allele compared to the wild type allele for number of viable spikelets $(P=0.43)$, seeds per spike $(P=0.91)$ nor seeds per spikelet $(P=0.96)$ (Table 4). In both sets of NILs the G2373A allele led to significant increases in TGW (Table 3); when combined to the non-significant effects on spike yield components this translates into an overall significant increase in the individual spike yield $(P<0.001)$. However, despite the positive effect being present in both $\mathrm{BC}_{2}(2.7 \%)$ and $\mathrm{BC}_{4}(9.6 \%)$ NILs, it was only significant in the $\mathrm{BC}_{4}$ NILs (Table 4).

\section{Distribution of grain morphometric parameters}

We further characterised the effect of the mutation by examining the distribution of grains across the different morphometric parameters. The individual values of all measured grains from BC-derived lines were used to generate box 
Fig. 4 Box and whisker plots of grain morphometric parameters of BC-derived lines in tetraploid wheat Kronos a and hexaploid wheat Paragon b, with the wild type GW2-A (grey) or G2373A gw2-A mutant allele (blue and green in tetraploid and hexaploid, respectively). Each row represents a separate experiment and the columns represents grain area (left), grain width (middle) and grain length (right). The left boundary of the box indicates the 25th percentile, the black line within the box marks the median (50th percentile), and the right boundary of the box indicates the 75 th percentile. The error bars (whiskers) on either side of the box indicate the 10th and 90th percentiles. The red line within the box marks the mean a

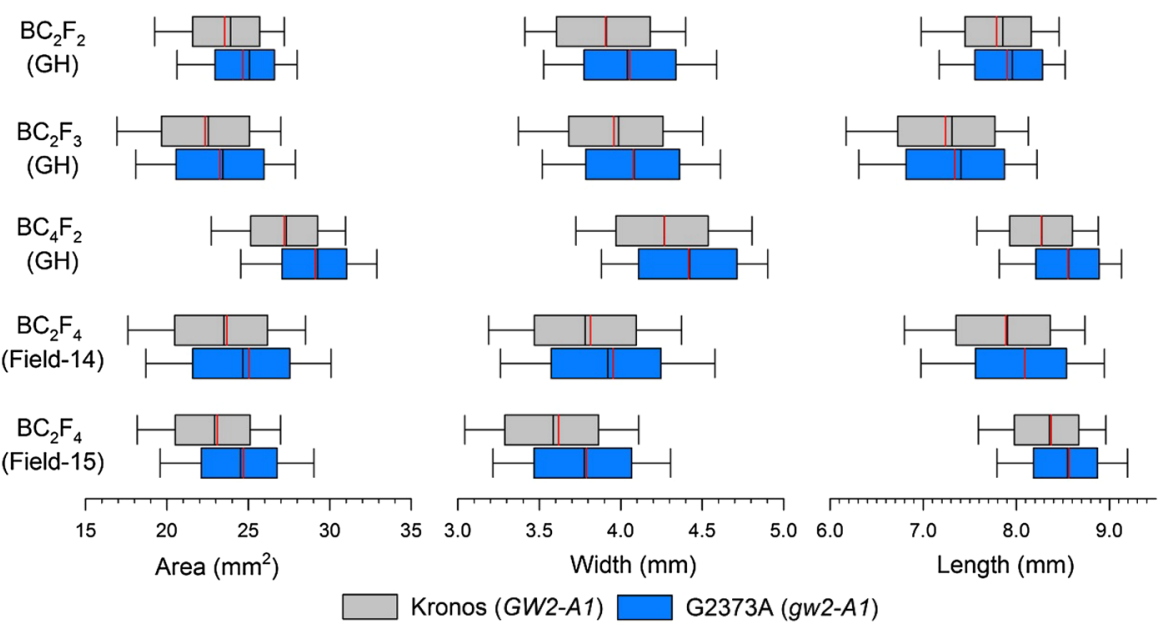

b

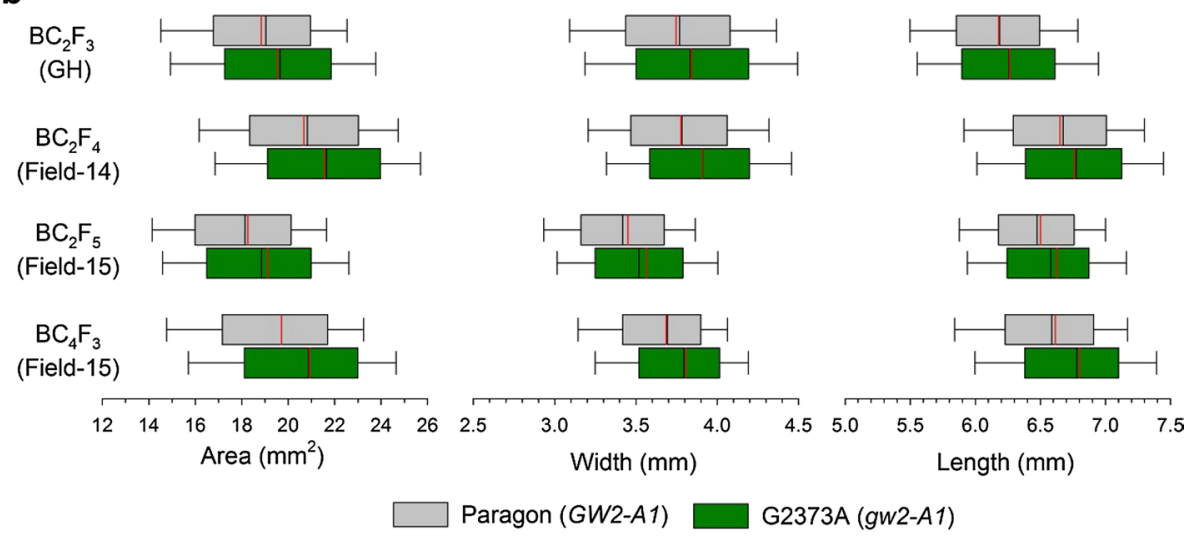

and whisker plots (Fig. 4). Across experiments, the G2373A mutation led to an increase in the median and the 5th, 10th, 25th, 75th, 90th, and 95th percentile values (Online Resource 3 ), in addition to the mean (Tables 2, 3). The mutation thus consistently increases the values of grains across different size categories, suggesting that the effect of the mutation is stable across the ear and within spikelets. This is reflected in the distribution shift for area, width and length in both tetraploid and hexaploid wheat across all experiments (Fig. 4).

\section{The G2373A mutation increases wheat carpel size}

We performed a developmental time course of carpel/grain size to assess when the differences in grain morphometric parameters were established. We sampled carpels/grains from field-grown $\mathrm{BC}_{2}$ Paragon NILs differing for the G2373A mutation across six time points: heading $(-5 \mathrm{dpa})$, anthesis $(0$ dpa), 3, 9, 16, and 23 dpa. We identified significant differences from carpel width $(6 \%)$ and length $(6 \%)$ at heading (Fig. 5a, b; $P<0.001)$. These differences were maintained across the time course for width, whereas for length the differences became less significant after $3 \mathrm{dpa}$ and were non-significant by the middle of grain fill ( $23 \mathrm{dpa}$ ). The increase in carpel/grain size also translated into increased grain dry weight; G2373A $\mathrm{BC}_{2}$ NILs had significantly heavier carpels and grains from heading ( $-5 \mathrm{dpa}$ ) onwards (Fig. 5c). Grain filling rates were generally higher in the G2373A NIL compared to the Paragon NIL, although these differences were only significant between 2 and 9 dpa ( 0.45 vs $0.38 \mathrm{mg}$ /day/grain, respectively; $P<0.01)$. Carpel/grain moisture content between NILs differed by less than $1 \%$ at each time-point $(P>0.05)$ suggesting that the effect of the G2373A mutation was not due to developmental differences but rather to an overall increase in size and weight. These results are consistent with the final harvest values of the same experimental units $\left(\mathrm{BC}_{2} 2015\right.$; Table 3$)$.

\section{Discussion}

\section{Is $T a G W 2-A 1$ a negative or positive regulator of grain size?}

In this study, we show that a splice acceptor site mutation in $T a G W 2-A 1$ leads to an increase in TGW in tetraploid 

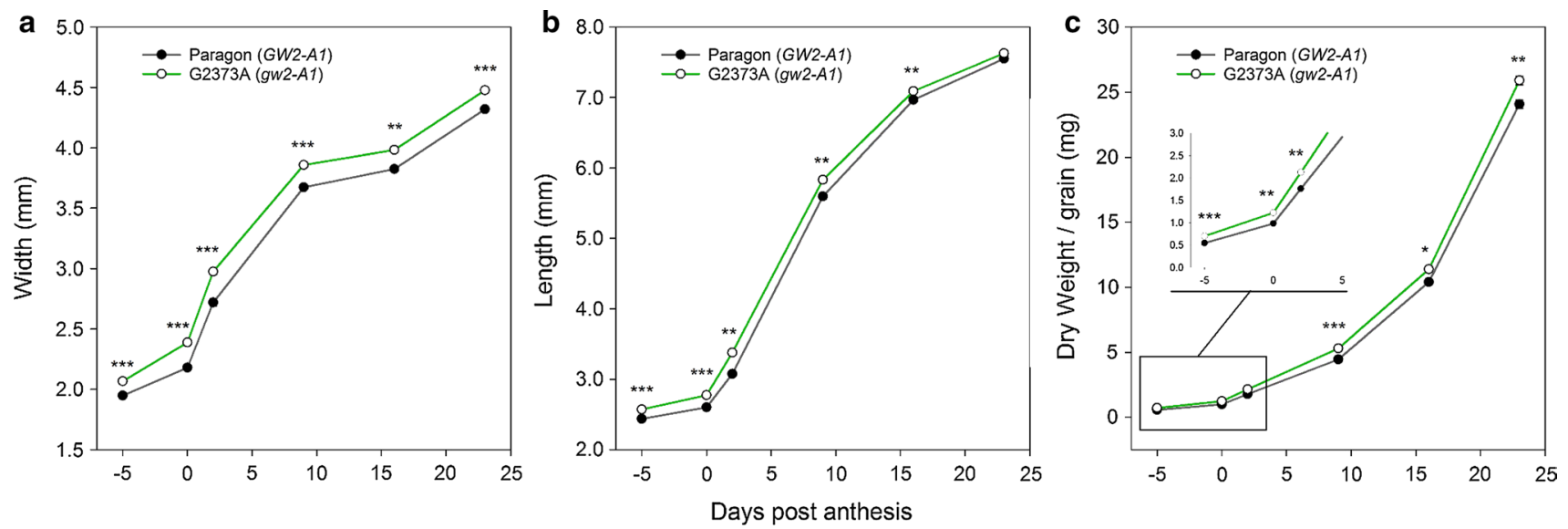

Fig. 5 Carpel/grain developmental time course of G2373A gw2-A1 NILs. Carpel/grain width (a), length (b), and dry weight (c) in Paragon $\mathrm{BC}_{2}$ NILs carrying either the Paragon wild type GW2-A (dark grey) or the G2373A gw2-A1 mutant allele (green). Samples were taken at heading [ -5 days post anthesis (dpa)], anthesis (0 dpa)

and hexaploid wheat. Our results are consistent with the rice $G W 2$ gene which acts as a negative regulator of grain weight and size (Song et al. 2007). These results are also in agreement with association studies in hexaploid wheat that have identified a negative association between TaGW2-A1 transcript abundance and grain weight and size (mainly width) (Hong et al. 2014; Qin et al. 2014; Song et al. 2007; Su et al. 2011). The negative effect of $T a G W 2-A 1$ on grain size has also been described in a traditional bi-parental mapping population (Yang et al. 2012). An allele with a single bp insertion was identified in the last exon of the gene leading to a premature termination codon in hexaploid landrace Lankaodali (328 amino acid peptide). When evaluated in a $\mathrm{F}_{2: 3}$ mapping population, the mutant $T a G W 2-A 1$ allele was associated with significantly increased TGW $(10.1 \%)$, grain width $(5.8 \%)$ and grain length $(2.4 \%)$ across three environments, compared to the wild type (full length) Chinese Spring allele (Yang et al. 2012). Our current study extends these results by showing that a novel gw2-Al mutant allele increases TGW, grain width and length in tetraploid durum wheat, as well as in hexaploid bread wheat. We developed near isogenic lines to account for background variation associated with the EMS mutagenesis and conducted 13 field and glasshouse experiments which showed that the gw2-A1 mutant allele significantly increases TGW, grain width and length by $6.6,2.8$, and $2.1 \%$, respectively. A detailed analysis of grain morphometric parameters showed that this effect was consistent across grains of all sizes, suggesting that the effect of the mutation is stable across the ear and within spikelets.

The role of TaGW2-Al on grain size came into question recently after a study showed that the simultaneous downregulation of the three $T a G W 2$ homoeologues by RNAi led and 3, 9, 16, and 23 dpa. Inset in (c) magnifies the first three time points to help visualise differences. Asterisks indicate significance of pairwise comparisons at each time point: ${ }^{*} P<0.05$; $* * P<0.01$; $* * * P<0.001$

to a $30 \%$ reduction in grain weight and an $11 \%$ decrease in grain width in hexaploid wheat (Bednarek et al. 2012). However, an equivalent study reported contrasting results; down-regulation of $T a G W 2$ homoeologues through RNAi led to an $18 \%$ increase in grain weight and a $5 \%$ increase in grain width in two independent transgenic lines (Hong et al. 2014). Despite the differences between these studies (genetic backgrounds, RNA trigger sequences, growing conditions, etc.) it is difficult to understand the conflicting nature of these results given our current knowledge of $T a G W 2$. Different functions of the three $T a G W 2$ homoeologues, subtle changes in their relative abundance within the transformed genotypes (Hong et al. 2014) and differing levels of down-regulation of the three homoeologues by the RNAi constructs could account for these inconsistencies. It is also possible that GW2 plays additional roles, and that a severe down-regulation of all homoeologues results in a general decrease of fitness that did not allow the plants to capitalize on the potential increase in grain size. We are currently generating gw2 null mutants combining loss-offunction mutations in all homoeologues to test this hypothesis. Even when considering the discrepant RNAi study, the accumulated association, genetic, mutant and transgenic evidence suggests that $T a G W 2-A 1$ functions as a negative regulator of grain weight and grain morphometric parameters in polyploid wheat.

These results suggest a possible conserved role of $G W 2$ (an E3 ubiquitin ligase) between wheat and rice, and this relationship seems also to extend into other plant species. The overexpression of the rice GW2 gene in Arabidopsis restricts seed (and organ) growth, whereas knockout of the GW2 homologue (Arabidopsis DA2 gene) leads to an increase in seed size (Xia et al. 2013). In maize, the 
GW2 homologue ZmGW2-CHR4 was shown to be associated with grain weight and width in an association panel and transcript levels were negatively correlated with grain width (Li et al. 2010). Our results on carpel/grain size development suggest that the wheat GW2 protein acts on maternal tissue before anthesis to restrict seed size. This is consistent with the role of Arabidopsis homologues DA1 and DA2 which affect cell proliferation in the integument (seed coat) (Li et al. 2008; Xia et al. 2013). Taken together, these results point to a conserved role of $G W 2$ homologues across plants and support the emerging concept that the ubiquitin pathway contributes to determine final seed size across multiple plant species (Li and Li 2015).

\section{Phenotypic effects of $G W 2$ mutants in wheat and rice}

The wheat G2373A allele results in the mis-splicing of the $T a G W 2-A 1$ transcript leading to two alternative protein forms. The predominant splicing results in a predicted 134 amino acid peptide with a premature truncation of the TaGW2-A1. This is analogous to the rice gw2 mutant allele which results in a 115 amino acid peptide (Song et al. 2007). The second, less frequent splicing variant results in a protein with a predicted wild type open reading frame, but which lacks three amino acid residues (EEQ). Despite these residues being located $3^{\prime}$ of the conserved RINGdomain, they are highly conserved across GW2 homologues in sequenced genomes including monocotyledonous and dicotyledonous species (Online Resource 4). This high degree of conservation suggests a potential functional role for these amino acids and a potential negative effect on GW2 protein function. Additional experiments would be required to test this hypothesis.

The wheat and rice gw2 mutations both affect grain size and shape, however, despite the use of similar germplasm ( $\mathrm{BC}_{2} \mathrm{~F}_{2}$ populations or more advanced backcross lines) the magnitude of the effect is almost eightfold higher in rice. Near isogenic lines carrying the rice gw2 mutant allele have $49.8 \%$ higher TGW and $26.2 \%$ wider grains compared to equivalent lines with the wild type $G W 2$ allele (Song et al. 2007). In tetraploid wheat, $\mathrm{BC}_{2}$ and $\mathrm{BC}_{4}$ near isogenic lines with the G2373A gw2-A1 allele have $6.2 \%$ higher TGW and $3.5 \%$ wider grains compared to the wild type allele. A similar effect is seen in $\mathrm{BC}_{2}$ NILs in hexaploid wheat (7.3\% higher TGW and $2.8 \%$ wider grains in the presence of $g w 2-A 1)$.

These results could be simply explained by functional redundancy in polyploid wheat: an $O s G W 2$ knockout mutant in rice generates a $g w 2$ null allele, whereas a TaGW2-A1 mutation only affects the dosage of the gene. The high amino acid conservation between TaGW2 homoeologues (97-98\%) and their similar expression pattern during grain development (Bednarek et al. 2012; Hong et al. 2014), suggests that the gw2-A1 mutant could lead to more subtle phenotypic variation compared to the equivalent rice mutant due to the functional complementation of the B- and D-genomes. The results from the RNAi study of Hong and co-workers (Hong et al. 2014) support this hypothesis since simultaneous down-regulation of all three TaGW2 homoeologues to $40 \%$ wild type levels leads to an increase in $18.1 \% \mathrm{TGW}$ and $5.4 \%$ wider grains in hexaploid wheat. However, given that these results are from a single experiment with unknown replicate number (Hong et al. 2014) and the contradictory results of Bednarek et al. (2012), caution must be used when interpreting and comparing the absolute values across studies. We cannot rule out that the G2373A splice variant missing three amino acids is able to provide some level of GW2-A1 activity which complements the other genomes. Alternatively, the potential for genetic improvement of grain weight and size through modification of TaGW2 could be more limited in wheat compared to rice. This could reflect the different morphology of maternal spikelet tissues between wheat and rice (Tashiro and Wardlaw 1989).

The side-by-side evaluation of complete $T a G W 2$ knockouts (and their factorial combinations) in different genetic backgrounds and environments will help establish putative dosage effects of $T a G W 2$. We have identified additional $T a G W 2-A 1$ and $T a G W 2-B 1$ truncation alleles in the tetraploid Kronos TILLING population and are currently developing the double $T a G W 2-A 1$ and $T a G W 2-B 1$ null mutant to test these hypotheses. A triple mutant in Paragon is also being developed with a TaGW2-D1 knockout allele. The study of this germplasm will reveal if larger genetic improvements of grain weight and size are achievable through the modification of $T a G W 2$ beyond those reported in the present study.

\section{Potential of the G2373A $g w 2-A 1$ allele in breeding}

The G2373A gw2-Al allele provides a significant increase in grain weight and size across glasshouse and field conditions in both tetraploid and hexaploid wheat. This increase is consistent across different grain size categories suggesting a stable effect across the length of the spike and within individual spikelets. It will now be important to evaluate if this effect translates into an equivalent increase in yield under field conditions, given the known compensations between yield components in wheat (Slafer et al. 1996). Our results from the $\mathrm{BC}_{2}$ and $\mathrm{BC}_{4}$ hexaploid NILs show that the mutant allele did not affect spikelet number, seeds per spike and seeds per spikelet in the UK in 2015. This suggests that the grain size effect can translate into overall yield, although additional experiments across multiple locations and genetic backgrounds will be needed to extend these conclusions beyond the genotype and environment 
tested in this study. From an agronomic point of view, the shift in grain size is also important since it reduces the proportion of small and light grains which can be lost during combine harvesting.

The Kronos and Paragon isogenic lines developed in this study are available to the wheat genetics and breeding communities through the JIC Germplasm Resources Unit (https://seedstor.ac.uk/; accession numbers W10281W10284) and the US National Small Grains Collection (PI675010-PI675015). We are also currently incorporating the allele into additional European winter wheat varieties and USA and CIMMYT spring wheat and durum lines. This material will help determine the potential of $g w 2-A 1$ in improving grain size and yield across environments. The development of the KASPar SNP marker should also help deploy the $g w 2-A 1$ allele into additional breeding programmes.

\section{Use of TILLING mutants to study quantitative variation in polyploids}

Understanding the relationship between homoeologues is particularly relevant for improving quantitative traits in wheat, since breeders can utilize allelic variation in each of the homoeologues separately or combined to modulate the desired trait response. The use of stable knockout alleles, such as the TILLING mutant identified in the present study, allows the evaluation of the relative contribution of individual homoeologues and the effects of double and triple mutant combinations (Avni et al. 2014; Botticella et al. 2011; Hazard et al. 2012, 2014; Slade et al. 2012). A relevant example on the use of TILLING mutants for this purpose is provided by work on a grain protein content QTL which was cloned in polyploid wheat (Uauy et al. 2006). The QTL was identified as a frame-shift mutation in the B-genome of a NAC transcription factor $(N A M-B 1)$ which led to a delay in senescence of 2 days and a decrease in protein content of 5-10\%. Simultaneous down-regulation of the different homoeologues by RNAi led to a much stronger phenotype in which senescence was delayed by more than 25 days and protein content was reduced by over $30 \%$. This broad phenotypic spectrum could be modulated through the use of individual nam TILLING mutants (Avni et al. 2014). nam-al/nam- $d l$ double mutants delayed senescence by 20-30 days compared to the wild type plants, whereas in single mutants this delay was between 5 and 10 days. An analogous phenotypic range for grain protein content was achieved through the use of double and single nam mutants.

Author contribution statement JS developed the tetraploid and hexaploid backcross populations used in this study, analysed the data and wrote the manuscript;
PS led the phenotypic assessments, and provided assistance with field trial preparation and glasshouse husbandry; JB conducted the developmental time course, qRT-PCR analysis and analysed the data; TCM conducted the TILLING screen of TaGW2-Al and identified the G2373A mutant allele; MB conducted the cDNA sequencing of $g w 2-A 1 ; \mathrm{AdB}$ and JD conducted the field trials in Davis; CU conceived the study, analysed the data and wrote the manuscript. All authors read and approved the final manuscript.

Acknowledgments This work was supported by grants $\mathrm{BB} /$ $\mathrm{J} 004588 / 1, \mathrm{BB} / \mathrm{J} 003557 / 1$ and BBS/E/J/000CA444 from the UK Biotechnology and Biological Sciences Research Council (BBSRC) to $\mathrm{CU}$ and an International Wheat Yield Partnership grant to $\mathrm{CU}$ and JD. JD acknowledges support from the USDA National Institute of Food and Agriculture Grant 2011-68002-30029 (TriticeaeCAP) and from the Howard Hughes Medical Institute. The authors would like to thank Hana Simkova and Jaroslav Dolezel (Institute of Experimental Biology, Olomouc, Czech Republic) for providing DNA of flow-sorted chromosome arms, Mr Raphaël Véquaud for technical assistance, the IWGSC for pre-publication access to wheat chromosome arm survey sequence, and the JIC Field Trials Horticultural services for technical support for glasshouse and field experiments.

\section{Compliance with ethical standards}

Conflict of interest The authors declare that they have no conflict of interest.

Open Access This article is distributed under the terms of the Creative Commons Attribution 4.0 International License (http://creativecommons.org/licenses/by/4.0/), which permits unrestricted use, distribution, and reproduction in any medium, provided you give appropriate credit to the original author(s) and the source, provide a link to the Creative Commons license, and indicate if changes were made.

\section{References}

Avni R, Zhao R, Pearce S, Jun Y, Uauy C, Tabbita F, Fahima T, Slade A, Dubcovsky J, Distelfeld A (2014) Functional characterization of GPC-1 genes in hexaploid wheat. Planta 239:313-324

Bednarek J, Boulaflous A, Girousse C, Ravel C, Tassy C, Barret P, Bouzidi MF, Mouzeyar S (2012) Down-regulation of the TaGW2 gene by RNA interference results in decreased grain size and weight in wheat. J Exp Bot 63:5945-5955

Borrill P, Adamski N, Uauy C (2015) Genomics as the key to unlocking the polyploid potential of wheat. New Phytol 208:1008-1022

Botticella E, Sestili F, Hernandez-Lopez A, Phillips A, Lafiandra D (2011) High resolution melting analysis for the detection of EMS induced mutations in wheat SbeIIa genes. BMC Plant Biol $11: 156$

Brenchley R, Spannagl M, Pfeifer M, Barker GLA, D'Amore R, Allen AM, McKenzie N, Kramer M, Kerhornou A, Bolser D, Kay S, Waite D, Trick M, Bancroft I, Gu Y, Huo N, Luo M-C, Sehgal S, Gill B, Kianian S, Anderson O, Kersey P, Dvorak J, McCombie WR, Hall A, Mayer KFX, Edwards KJ, Bevan MW, Hall N (2012) Analysis of the bread wheat genome using wholegenome shotgun sequencing. Nature 491:705-710 
Breseghello F, Sorrells ME (2007) QTL analysis of kernel size and shape in two hexaploid wheat mapping populations. Field Crops Res 101:172-179

Chen A, Dubcovsky J (2012) Wheat TILLING mutants show that the vernalization gene $V R N 1$ down-regulates the flowering repressor $V R N 2$ in leaves but is not essential for flowering. PLoS Genet 8:e1003134

Chen A, Li C, Hu W, Lau MY, Lin H, Rockwell NC, Martin SS, Jernstedt JA, Lagarias JC, Dubcovsky J (2014) PHYTOCHROME C plays a major role in the acceleration of wheat flowering under long-day photoperiod. Proc Natl Acad Sci 111:10037-10044

Fan C, Xing Y, Mao H, Lu T, Han B, Xu C, Li X, Zhang Q (2006) GS3, a major QTL for grain length and weight and minor QTL for grain width and thickness in rice, encodes a putative transmembrane protein. Theor Appl Genet 112:1164-1171

FAOSTAT (2015) http://faostat3.fao.org/home

Fu D, Uauy C, Distelfeld A, Blechl A, Epstein L, Chen X, Sela H, Fahima T, Dubcovsky J (2009) A kinase-START gene confers temperature-dependent resistance to wheat stripe rust. Science 323:1357-1360

Hazard B, Zhang X, Colasuonno P, Uauy C, Beckles DM, Dubcovsky J (2012) Induced mutations in the Starch Branching Enzyme II (SBEII) genes increase amylose and resistant starch content in durum wheat. Crop Sci 52:1754-1766

Hazard B, Zhang X, Naemeh M, Dubcovsky J (2014) Registration of durum wheat germplasm lines with combined mutations in SbeIIa and SbeIIb genes conferring increased amylose and resistant starch. J Plant Regist 8:334-338

Hong Y, Chen L, Du L-p, Su Z, Wang J, Ye X, Qi L, Zhang Z (2014) Transcript suppression of TaGW2 increased grain width and weight in bread wheat. Functional and Integrative Genomics:1-9

Huang X, Qian Q, Liu Z, Sun H, He S, Luo D, Xia G, Chu C, Li J, Fu X (2009) Natural variation at the DEP1 locus enhances grain yield in rice. Nat Genet 41:494-497

IWGSC (2014) A chromosome-based draft sequence of the hexaploid bread wheat (Triticum aestivum) genome. Science 345

Jiang W-B, Huang H-Y, Hu Y-W, Zhu S-W, Wang Z-Y, Lin W-H (2013) Brassinosteroid regulates seed size and shape in Arabidopsis. Plant Physiol 162:1965-1977

Krasileva K, Buffalo V, Bailey P, Pearce S, Ayling S, Tabbita F, Soria M, Wang S, Consortium I, Akhunov E, Uauy C, Dubcovsky J (2013) Separating homeologs by phasing in the tetraploid wheat transcriptome. Genome Biol 14:R66

Kuchel H, Williams KJ, Langridge P, Eagles HA, Jefferies SP (2007) Genetic dissection of grain yield in bread wheat. I. QTL analysis. Theor Appl Genet 115:1029-1041

Lawrence RJ, Pikaard CS (2003) Transgene-induced RNA interference: a strategy for overcoming gene redundancy in polyploids to generate loss-of-function mutations. Plant J 36:114-121

Li N, Li Y (2015) Maternal control of seed size in plants. J Exp Bot 66:1087-1097

Li Y, Zheng L, Corke F, Smith C, Bevan MW (2008) Control of final seed and organ size by the DAl gene family in Arabidopsis thaliana. Genes Dev 22:1331-1336

Li Q, Li L, Yang X, Warburton M, Bai G, Dai J, Li J, Yan J (2010) Relationship, evolutionary fate and function of two maize coorthologs of rice $G W 2$ associated with kernel size and weight. BMC Plant Biol 10:143

Maphosa L, Langridge P, Taylor H, Parent B, Emebiri L, Kuchel H, Reynolds M, Chalmers K, Okada A, Edwards J, Mather D (2014) Genetic control of grain yield and grain physical characteristics in a bread wheat population grown under a range of environmental conditions. Theor Appl Genet 127:1607-1624

McCallum CM, Comai L, Greene EA, Henikoff S (2000) Targeting induced local lesions in genomes (TILLING) for plant functional genomics. Plant Physiol 123:439-442
Pallotta M, Warner P, Fox R, Kuchel H, Jefferies S, Langridge P (2003) Marker assisted wheat breeding in the southern region of Australia. Proc 10th Int Wheat Genet Symp, Paestum, Italy, pp $1-6$

Qin L, Hao C, Hou J, Wang Y, Li T, Wang L, Ma Z, Zhang X (2014) Homologous haplotypes, expression, genetic effects and geographic distribution of the wheat yield gene $T a G W 2$. BMC Plant Biol 14:107

Ray DK, Mueller ND, West PC, Foley JA (2013) Yield trends are insufficient to double global crop production by 2050. PLoS One 8:e66428

Rustgi S, Shafqat MN, Kumar N, Baenziger PS, Ali ML, Dweikat I, Campbell BT, Gill KS (2013) Genetic dissection of yield and its component traits using high-density composite map of wheat chromosome 3A: bridging gaps between QTLs and underlying genes. PLoS One 8:e70526

Schmittgen TD, Livak KJ (2008) Analyzing real-time PCR data by the comparative CT method. Nat Protocols 3:1101-1108

Simmonds J, Scott P, Leverington-Waite M, Turner AS, Brinton J, Korzun V, Snape J, Uauy C (2014) Identification and independent validation of a stable yield and thousand grain weight QTL on chromosome 6A of hexaploid wheat (Triticum aestivum L.). BMC Plant Biol 14:191

Slade A, McGuire C, Loeffler D, Mullenberg J, Skinner W, Fazio G, Holm A, Brandt K, Steine M, Goodstal J, Knauf V (2012) Development of high amylose wheat through TILLING. BMC Plant Biol 12:69

Slafer G, Calderini D, Miralles D (1996) Yield components and compensation in wheat: opportunities for further increasing yield potential. Increasing yield potential in wheat: Breaking the Barriers:101-133

Song X-J, Huang W, Shi M, Zhu M-Z, Lin H-X (2007) A QTL for rice grain width and weight encodes a previously unknown RING-type E3 ubiquitin ligase. Nat Genet 39:623-630

Su Z, Hao C, Wang L, Dong Y, Zhang X (2011) Identification and development of a functional marker of $T a G W 2$ associated with grain weight in bread wheat (Triticum aestivum L.). Theor Appl Genet 122:211-223

Sun X-Y, Wu K, Zhao Y, Kong F-M, Han G-Z, Jiang H-M, Huang X-J, Li R-J, Wang H-G, Li S-S (2009) QTL analysis of kernel shape and weight using recombinant inbred lines in wheat. Euphytica 165:615-624

Tashiro T, Wardlaw IF (1989) A comparison of the effect of high temperature on grain development in wheat and rice. Ann Bot 64:59-65

Trick M, Adamski N, Mugford S, Jiang C-C, Febrer M, Uauy C (2012) Combining SNP discovery from next-generation sequencing data with bulked segregant analysis (BSA) to fine-map genes in polyploid wheat. BMC Plant Biol 12:14

Uauy C, Distelfeld A, Fahima T, Blechl A, Dubcovsky J (2006) A NAC gene regulating senescence improves grain protein, zinc, and iron content in wheat. Science 314:1298-1301

Uauy C, Paraiso F, Colasuonno P, Tran R, Tsai H, Berardi S, Comai L, Dubcovsky J (2009) A modified TILLING approach to detect induced mutations in tetraploid and hexaploid wheat. BMC Plant Biol 9:115

Vrána J, Kubaláková M, Simková H, Č́íhalíkovái J, Lysák MA, Dolezel J (2000) Flow sorting of mitotic chromosomes in common wheat (Triticum aestivum L.). Genetics 156:2033-2041

Weng J, Gu S, Wan X, Gao H, Guo T, Su N, Lei C, Zhang X, Cheng Z, Guo X (2008) Isolation and initial characterization of GW5, a major QTL associated with rice grain width and weight. Cell Res 18:1199-1209

Williams K, Sorrells ME (2014) Three-dimensional seed size and shape QTL in hexaploid wheat (Triticum aestivum L.) populations. Crop Sci 54:98-110 
Xia T, Li N, Dumenil J, Li J, Kamenski A, Bevan MW, Gao F, Li Y (2013) The ubiquitin receptor DA1 interacts with the E3 ubiquitin ligase DA2 to regulate seed and organ size in Arabidopsis. The Plant Cell Online 25:3347-3359

Xing Y, Zhang Q (2010) Genetic and molecular bases of rice yield. Annu Rev Plant Biol 61:421-442

Yang Z, Bai Z, Li X, Wang P, Wu Q, Yang L, Li L, Li X (2012) SNP identification and allelic-specific PCR markers development for $T a G W 2$, a gene linked to wheat kernel weight. Theor Appl Genet 125:1057-1068
Zhang L-Y, Liu D-C, Guo X-L, Yang W-L, Sun J-Z, Wang D-W, Zhang A (2010) Genomic distribution of quantitative trait loci for yield and yield-related traits in common wheat. J Integr Plant Biol 52:996-1007

Zhang X, Chen J, Shi C, Chen J, Zheng F, Tian J (2013) Function of TaGW2-6A and its effect on grain weight in wheat (Triticum aestivum L.). Euphytica 192:347-357 